\title{
Liver-directed therapy for hepatocellular carcinoma
}

\author{
Cathal O'Leary ${ }^{1}$, Mary Mahler $^{2}$, Michael C. Soulen ${ }^{1}$ \\ ${ }^{1}$ Department of Radiology, University of Pennsylvania, Philadelphia, PA, USA; ${ }^{2}$ Department of Medical Oncology, University of Toronto, Toronto, \\ Ontario, Canada \\ Contributions: (I) Conception and design: All authors; (II) Administrative support: None; (III) Provision of study materials or patients: None; (IV) \\ Collection and assembly of data: All authors; (V) Data analysis and interpretation: All authors; (VI) Manuscript writing: All authors; (VII) Final \\ approval of manuscript: All authors. \\ Correspondence to: Michael C. Soulen, MD, FSIR, FCIRSE. Department of Radiology, 3400 Spruce St, Philadelphia, PA 19104, USA. \\ Email: michael.soulen@pennmedicine.upenn.edu.
}

\begin{abstract}
The high mortality rate for hepatocellular carcinoma (HCC) relative to its prevalence underscores the need for curative-intent therapies. Multidisciplinary treatment decisions are required to craft optimal treatment strategies considering tumor size, location and underlying liver cirrhosis. Surgical resection of anatomically limited tumors with adequate hepatic reserve provides long-term survival in more than half of patients and remains a standard first-line therapy. Eligibility for resection among newly diagnosed patients is low and recurrences in the remaining cirrhotic liver are common. Transplantation offers a higher chance of cure. Long wait times for the limited door pool require neoadjuvant loco-regional therapies to maintain transplant eligibility. Image-guided therapies such as ablation and embolization have an established role as primary or neoadjuvant preparing patients for curative treatment. Percutaneous ablation in appropriately selected patients offers long-term survival similar to resection. New and evolving techniques such as stereotactic body radiotherapy (SBRT), radiation segmentectomy and lobectomy, and combination therapies employing both trans-arterial and ablative approaches show promise for curative-intent treatment but require further prospective data before they can be integrated into treatment algorithms. For palliativeintent therapy, conventional trans-arterial chemoembolization with lipiodol-based emulsions remains the only technique supported by clinical trials. Newer platforms such as drug-eluting embolics failed to improve survival over bland embolization in randomized trials and showed increased hepatobiliary toxicity. Transarterial radioembolization offers similar overall survival (OS) to transarterial chemoembolization (TACE) and non-inferiority to sorafenib in meta-analyses. The more favorable clinical toxicity profile makes it an appealing technique for patients willing to accept the longer time to response.
\end{abstract}

Keywords: Hepatocellular carcinoma (HCC); ablation; chemoembolization; radioembolization; liver transplantation

Submitted Feb 01, 2020. Accepted for publication Apr 29, 2020.

doi: $10.21037 /$ cco-20-51

View this article at: http://dx.doi.org/10.21037/cco-20-51

\section{Introduction}

Hepatocellular carcinoma (HCC) is the 6th most common tumor occurring internationally and 3rd leading cause of cancer deaths worldwide (1). Its prevalence is closely linked to that of chronic liver disease with most cases occurring in Asia and sub-Saharan Africa, where the predominant cause is the hepatitis B virus. Western populations have a higher fraction of hepatitis $\mathrm{C}$ cirrhosis, followed by alcohol and non-alcoholic steatohepatitis (NASH) cirrhosis (2). An important concept is that the majority of patients with HCC have two diseases, HCC and the underlying liver disease that predisposes to new tumor formation. Staging and treatments plans consider both.

The Barcelona Clinic Liver Cancer (BCLC) classification is extensively validated and endorsed by both the European 
Association for the Study of the Liver (EASL) panel of experts and the American Association for the Study of Liver Diseases (AASLD) guidelines $(3,4)$. BCLC uses tumor size and number, Child-Pugh score, and Eastern Cooperative Oncology Group (ECOG) performance status to stratify among treatment strategies. This differs from standard cancer staging systems in that a change in ECOG PS from 0 to 1 changes the "stage" and treatment recommendations, while conversely patients with and without metastatic disease can be the same "stage".

An alternative classification is the Hong Kong Liver Cancer staging system, which combines performance status, tumor size and number, Childs-Pugh score, intrahepatic venous invasion, and extrahepatic venous invasion or metastasis to allocate patients among liverdirected therapies, systemic therapy, or supportive care (5). This model more aggressively employs surgery and chemoembolization in BCLC intermediate and advanced stages (BCLC B and C), including patients with liverlimited portal venous invasion, with improved survival over that predicted in the BCLC model.

One third of patients with a new diagnosis of $\mathrm{HCC}$ will be eligible for curative intent therapy (6). These approaches are typically limited to patients with early-stage HCC (BCLC A), a solitary lesion or up to three nodules that are less than $3 \mathrm{~cm}$ in diameter, with preserved liver function and without macrovascular invasion or extrahepatic spread; or very early-stage HCC (BCLC 0), a subgroup with single tumors $\leq 2 \mathrm{~cm}$ in diameter. Intermediate-stage HCC patients (BCLC B) have larger or more numerous tumors without vascular invasion or extrahepatic spread. They may be considered for downstaging to liver transplant (7). More advanced (BCLC C \& D) patients have an array of systemic therapy options available that will not be discussed further in this article. This review will focus on curative intent therapies (ablation, surgical resection and transplantation) and the new or established trans-arterial liver-directed treatments.

\section{Early stage HCC (BCLC 0 \& A)}

Ablation, resection, or transplantation are the curative intent therapies offered to BCLC A patients $(3,4)$. These approaches provide a 5 -year survival rate of $50-80 \%$ $(8,9)$. Recurrence is common for patients with HCC, complicating up to $70 \%$ of patients after 5 years $(1,10)$. Liver transplant is the optimal treatment of both the tumor and underlying carcinogenic liver disease but is hampered by a limited organ supply. Surgical resection remains the $1^{\text {st }}$ line treatment for localized tumors in both cirrhotic and non-cirrhotic patients according to the latest EASL, AASLD and National Comprehensive Cancer Network (NCCN) guidelines $(3,4,11)$. However, only $5-10 \%$ of HCC patients will present with resectable tumors (1). Ablation has gained recognition in consensus guidelines for treatment of unresectable early stage tumors. It has the added benefit of maintaining maximal liver parenchyma. A variety of patient factors, regional expertise and patient preference determine the treatment of choice.

\section{Curative intent locoregional therapy for single HCC}

Anatomic hepatectomy attempts to remove potential tumor cells along portal venous tributaries within the involved liver segment, an approach that has been shown to improve overall survival (OS). With modern surgical techniques, it offers low perioperative morbidity and 5 -year survival rates nearing $70 \%$ in appropriately selected patients (3). The resection of segmental anatomy is of importance due to the microsatellites that form with HCC (12). Venous microvascular invasion occurs in a size dependent fashion with increasing number of microsatellites seen with larger tumors $(12,13)$. Resection allows for complete pathologic staging and potentially pre-emptive transplant for patients at high risk of recurrence post resection (14). The issue of resectability is key and revolves around tumor, liver and patient factors with guidelines a goal overall mortality of $3 \%$ and morbidity of $30 \%$ (3). In patients who are not resection candidates, ablation or transplantation are used (4).

Percutaneous ablation includes several methods of chemical or energy-based tumor destruction via percutaneous, open or laparoscopic approaches. The most common, heat-based techniques use electrical current (radiofrequency ablation-RFA) or electromagnetic energy (microwave ablation-MWA) to induce coagulative necrosis within the tumor. They have superior response and local recurrence (LR) rates compared to percutaneous ethanol injection, an earlier method of ablation $(4,15,16)$. The heat-based techniques allow for the necrosis of a rind of peritumoral liver that may eliminate small tumoral satellites. MWA has advantages of a shorter delivery time and multiple probe use that create larger, more predictable ablation zone than RFA.

Advocates argue for thermal ablation to be considered $1^{\text {st }}$ line therapy for all candidates, citing its minimally invasive nature, preservation of liver parenchyma and low 
complication rate. It is limited by anatomic considerations, e.g., adjacent vessels and organs, and inadequate tumor necrosis for tumors $>3 \mathrm{~cm}$. Meta-analyses of RCT data have failed to show a conclusive difference in all-cause mortality between surgical resection and RFA in single, resectable tumors, excluding data from a 2010 RCT that utilized a single RFA probe, an approach that has since been abandoned due to concerns for incomplete ablation (16-18). Individual RCT data has not shown a significant difference in overall or disease-free survival for RFA and resection (19-22). A Cochrane review of 4 RCTs and 574 patients showed equivalent all-cause mortality [hazard ratio (HR) 0.80, 95\% CI: 0.6-1.08] (16). Although heterogeneity in reporting definitions limited grouped analysis of complications, both recent meta-analyses found a higher rate in the resection group $(16,17)$. The adjusted rate of serious adverse events was 11.3 per 100 participants for resection and 1.6 per 100 participants for ablation in two RCTs of 391 patients [rate ratio 7.02, 95\% CI: 2.29-21.46]. Both AASLD and NCCN consider resection as $1^{\text {st }}$ line, while EASL guidelines allow for the use of RFA in surgical candidates, if in favorable locations $(3,4,11)$. Many centers consider a complimentary approach to deciding between resection and ablation; with tumor size, location and patient comorbidities as the main considerations.

\section{Size $<2 \mathrm{~cm} / \mathrm{BCLC} 0$ patients}

An important subgroup to consider are the very-early stage, BCLC 0, patients with a solitary small nodule, $<2 \mathrm{~cm}$ in diameter, Child-Pugh class A disease, and no signs of microvascular invasion and dissemination. Very early stage tumors have such promising outcomes from curative intent treatment, both ablation and surgery, that they should not be listed for transplant initially. Ablation and resection provide complete response rates of $>97 \%$ and 5 -year survival rate of $>75 \%$ (23-25). Retrospective studies have suggested an OS benefit of resection compared to thermal ablation in this subgroup (26). However, available RCT data for this population, either primary or subgroup data, found no significant difference between RFA and resection in diseasefree survival (DFS), recurrence-free survival (RFS) or OS (19-21). A 2017 RCT compared ablation and resection for the very early subgroup $(n=55)$ and found no significant difference in either OS or disease free survival; with 1-, 3 -, 5- and 10 -year OS rates of $100 \%, 93 \%, 76 \%$ and $52 \%$ respectively in the resection group, compared with $100 \%$, $89 \%, 69 \%$ and $59 \%$ in the RFA group $(\mathrm{P}=0.950)(19)$. The
$1-, 3-, 5-$ and 10-year disease-free survival rates were $83 \%$, $66 \%, 52 \%$ and $35 \%$ respectively in the resection group, and $77 \%, 62 \%, 46 \%$ and $39 \%$ in the RFA group ( $\mathrm{P}=0.896)(19)$. This makes intuitive sense, as the small tumor size would have the lowest likelihood of microsatellite formation.

\section{Size $>3 \mathrm{~cm}$}

For tumors larger than $3 \mathrm{~cm}$, size is not a limitation to surgical resection if the tumor remains well circumscribed and without vascular invasion. Patients with larger tumors within resectability criteria are self-selected as more indolent, less aggressive tumors. Concerns exist over the ability to ablate tumors over $3 \mathrm{~cm}$ in diameter due to the larger ablation zones required to destroy the tumor and produce the $5 \mathrm{~mm}$ ablation margin required to encompass any adjacent microsatellites (27). Within the RCTs comparing resection and RFA, all patients were surgical candidates with single tumors $<4-5 \mathrm{~cm}(18-22)$. As previously mentioned, a 2010 RCT that used a single RFA electrode found improved RFS and OS rates with resection for patients with larger sized tumors, with 5 -year overall recurrence of $63.48 \%$ for RFA and $41.74 \%$ for resection $(\mathrm{P}=0.024)$ (18). The remaining RCTs found no significant difference between OS or DFS/RFS outcomes (19-22).

New techniques such as MWA and thermal ablation combined with transarterial chemoembolization (TACE) attempt to create a larger, more predictable treatment areas. MWA is increasing in popularity due to its speed and decreased impact from the heat sink effect. A 2016 metaanalysis comparing MWA and RFA showed equivalent complete ablation and OS outcomes but a significant improvement in LR rates for MWA (OR 0.48, 95\% CI: 0.24-0.89, $\mathrm{P}=0.02)(25)$. This significant difference in $\mathrm{LR}$ was not reproduced in a 2019 meta-analysis by Tan et al. (OR 0.85, 95\% CI: 0.35-2.10, $\mathrm{P}=0.73$ ) (23). Their analysis of RCT data showed no significant difference between percutaneous MWA and RFA in complete ablation (OR 0.85, 95\% CI: 0.41-1.79, $\mathrm{P}=0.67$ ), LR rates (OR 1.47, 95\% CI: $0.45-4.84, \mathrm{P}=0.52$ ) or three-year OS (OR 0.68, 95\% CI: $0.34-1.37, \mathrm{P}=0.28$ ) (23). The authors suspected the heterogeneity in available MWA technology, and the smaller size of tumors studied to date failed to show the full advantages of MWA. When comparing MWA directly to hepatic resection, a 2019 meta-analysis of both HCC and liver metastases, including 1 RCT of 90 patients with HCC, found a significantly higher recurrence risk for MWA (RR 2.49; 95\% CI: 1.19-5.22; $\mathrm{P}=0.016$ ) with less major 
complications $(\mathrm{RR}=0.31 ; \mathrm{P}<0.001)(28)$. RFA has a more established evidence base and safety profile. EASL, AASLD and NCCN guidelines each remark that MWA shows promising early results but that larger studies are required for safety and efficacy data $(3,4,11)$.

Combining TACE with ablation creates synergy by decreasing blood flow in the ablation zone while taking advantage of the synergy between doxorubicin and hyperthermia in the large periablational zone to maximize the volume of tumor necrosis. This reduces the possibility of microsatellite survival, recurrence and results in better OS than either treatment alone. A 2016 meta-analysis comparing combination with RFA monotherapy, which included 417 patients in 3 RCTs and a majority of tumors $\geq 3 \mathrm{~cm}$, found that combination therapy had an improved RFS rate with 1-, 3-, and 4-year rates of 71.3-79.4\%, 48.8$60.6 \%$, and $36.6-54.8 \%$ for TACE+RFA, and $66.7-74.3 \%$, $29.7-44.2 \%, 29.7-38.9 \%$ for RFA alone (HR $=0.55,95 \%$ CI: $0.40-0.76, \mathrm{P}<0.001)$. There was no significant increase in major complications, which occurred in 7/249 TACE+RFA patients and 6/249 RFA alone patients $(\mathrm{OR}=1.17$, 95\% CI: 0.39-3.55, $\mathrm{P}=0.78)$ (29). This was consistent with prior meta-analyses (30,31). A 2018 meta-analysis comparing TACE and RFA with resection for patients within Milan criteria found that TACE and RFA had an improved 1-year survival rate $(\mathrm{OR}=0.50, \mathrm{P}=0.009)$ and major complication rate (OR 1.88, $\mathrm{P}=0.02)$ compared to surgical resection (32). The OS benefit did not continue at 3 or 5 years and there was no advantage in recurrence free survival at any timepoint. Fewer studies have evaluated TACE and MWA. Similar results have been found in small RCTs evaluating TACE and MWA vs. MWA alone (33,34). Early cohort studies have examined its effect in larger tumors (max diameter up to $10 \mathrm{~cm}$ ) with results similar to surgical resection (35). Currently, guidelines recommend resection for surgical candidates with tumors larger than $2-3 \mathrm{~cm}$ and further prospective data on MWA and combination therapy is required to prove their suspected advantages and integrate them into the treatment paradigm $(4,11)$.

\section{Location}

Perivascular, perihilar and subcapsular locations can limit ablation effectiveness due to concerns of treatment efficacy and collateral damage to the gastrointestinal tract or biliary tree (36). Perivascular tumors (vessels $>3 \mathrm{~mm}$ in diameter) suffer from the heat-sink effect; heat lost by cooling of ablated tissue by adjacent large vessels $(37,38)$. MWA has a faster heating time that has been shown to overcome the heat sink effect in animal models (39). Clinical data comparing it with RFA is limited in proving this point, possibly due to difficulty comparing retrospective data on tumor location (23). There are differences in what physicians consider 'unable to ablate', with techniques ranging from laparoscopic ablation to occlusion balloon use in hepatic or portal venous branches trialed to safely ablate tumors in difficult locations (40). Recent large studies have shown the OS, local tumor progression and major complications are not significantly different regardless of the technique used (4). Local expertise in these more advanced techniques is an important consideration when choosing the best treatment modality. Importantly, percutaneous ethanol injection remains an option for small HCCs unsuitable for thermal ablation due to proximity to vital structures, subcapsular location, coagulopathy, or ascites.

\section{Comorbidities}

The most relevant and frequently encountered comorbidities are liver cirrhosis, with particular attention paid to the degree of portal hypertension. Portal hypertension has been described as the single biggest predictor of a poor outcome after surgery; predicting a higher risk of postoperative clinical decompensation and worse 3 - and 5 -year survival (41). EASL and AASLD guidelines recommend resection for Child-Pugh A patients only, while NCCN guidelines allow a provision for certain cases of well selected Child-Pugh B cirrhosis patients $(3,4,11)$. This well selected group refers to patients without portal hypertension, with a platelet count $>100,000$, absence of varices and a hepatic venous gradient $<10 \mathrm{mmHg}$; approximately $10 \%$ of all HCC (1). This leaves a large cohort at high risk from complications for whom ablation or transplant should be considered.

A second important consideration is that adequate functional reserve of at least $20 \%$ of normal liver and 30 $40 \%$ for patients with Child-Pugh Class A cirrhosis is required for good outcomes of resection $(3,11)$. Portal vein embolization is an accepted technique in patients whom the estimated future liver remnant (FLR) is too small to allow safe resection, usually prior to right hepatectomy (42). Portal vein embolization induces preoperative hypertrophy of the contralateral lobe at a cost of minimal derangement of liver function. Time must be allowed for regeneration, which occurs more slowly in damaged livers. A recommended minimum of 6 weeks should be allowed for 
hypertrophy, with studies showing that 1-2 months results in adequate results (43). This can be considered a test of the regenerative capacity of the liver, and failure to hypertrophy are an indication that the liver function is worse than it appears. Tumor progression is of concern during the hypertrophy period, particularly since the rate of tumor growth has been shown to increase compared to prior to PVE $(42,44)$. TACE and PVE combinations have been used to maintain tumor control during this period.

Multiple single-center retrospective studies have evaluated radiation lobectomy in HCC patients who are otherwise candidates for resection but have a small FLR (45). This involves performing unilobar (right lobe) radioembolization with a parenchymal dose of 80-150 Gy (45). The theorized mechanism is that of slow, controlled diversion of portal venous flow into the contralateral lobe due to atrophy of the treated lobe (45). Volumetric changes are slower than PVE, taking approximately 6-9 months for maximal effect $(45,46)$. To date, retrospective cohort data has shown excellent tumor control pending resection, which usually occurs after 3 months (45). This technique shows promise as a method of maintaining tumor control while awaiting resection, an important method of avoiding adding patients to the transplant waitlist.

\section{Newer approaches}

\section{External beam radiotherapy}

The use of radiotherapy for treating small hepatic lesions has been limited by concerns of efficacy and safety, in particular the risk of radiation-induced liver disease (RILD) to the normal liver parenchyma. Newer focused radiation delivery fields used in $3 \mathrm{D}$-conformal radiotherapy (3D-CRT), intensity modulated radiotherapy (IMRT), and stereotactic body radiotherapy (SBRT/SABR) have made it possible to improve precision in radiation delivery (47). This has led to growing interest on the use of EBRT at varying stages of HCC treatment.

EBRT has advantages over ablation in high-risk locations such as central lesions, below the diaphragm and those adjacent to the biliary system (47). Based on a retrospective analysis of 224 patients with inoperable, non-metastatic HCC, Wahl et al. suggest that SBRT can improve time to progression for lesions $>2 \mathrm{~cm}$ with similar rates of complications (48). They found overall 2-year freedom from local progression (FFLP) rates of $80.2 \%$ for RFA and $83.8 \%$ for SBRT, but RFA was significantly worse FFLP when the subgroup of patients with tumors $>2 \mathrm{~cm}$ was analyzed (HR, 2.35; 95\% CI: 1.17-9.62; $\mathrm{P}=0.025$ ) (48). This is at odds with a propensity matched retrospective analysis of the SEER database, which found that OS was equivalent between SBRT and ablation therapy (all types, of which $70 \%$ were 'heat-based') (49). A subgroup analysis of tumors $<3 \mathrm{~cm}$ found an OS of 37 months for ablation and 47 months for EBRT $(\mathrm{P}=0.508)$, while ablation therapy had a significant $O S$ benefit for tumors ranging $3-5 \mathrm{~cm}$ (20 vs. 16 months, $\mathrm{P} \leq 0.001)$ and $>5 \mathrm{~cm}(25$ vs. 9 months, $\mathrm{P} \leq 0.001$ ) (49). Well conducted, large prospective trial data is required to establish the position of EBRT in the treatment of early HCC. NCCN and AASLD guidelines consider EBRT a safe alternative when ablation or resection are contraindicated in patients with BCLC A disease, however, EASL guidelines consider the evidence base lacking for a recommendation.

\section{Transarterial radiation segmentectomy}

Transarterial radioembolization (TARE) uses minimally embolic microspheres loaded with Ytrrium-90, a $\beta$-emitting isotope, to deliver selective internal radiation to the tumor. It has a more established role in intermediate and advanced stages of disease, as will be discussed late in this article $(3,50)$. Firstly, we would like to address the novel approach of curative radiation segmentectomy for early stage disease (51). Explant pathologic data has shown that higher doses of radiotherapy, applied more focally, result in complete pathologic necrosis in approximately two thirds of HCC patients (46). This has stimulated interest in TARE as a curative intent approach for patients with BCLC A disease and solitary tumors $<5 \mathrm{~cm}$. Lewandowski et al. delivered doses $>190$ Gy to 1-2 segments in patients with unresectable HCC deemed unsafe for ablation (51). This group of 70 patients had $71-90 \%$ criteria-dependent initial response rate, time to progression of 2.4 years and OS of $55 \%$ at 5 years (51). The authors cite these rates of local tumor control, prolonged time to progression and OS comparable to ablation, resection and transplant as reasons to consider radiation segmentectomy curative for BCLC stage 0 or A patients with solitary tumors $<5 \mathrm{~cm}$. While these are promising results in a single retrospective cohort, prospective validation is required at other centers.

\section{Curative intent locoregional therapy for multifocal HCC}

Multifocal HCC is present at diagnosis in $35-40 \%$ of 
patients. Transplant is the preferred curative treatment for the patients that remain within BCLC A criteria $(3,4)$. Those with comorbidities preventing transplant are considered for ablation. Few studies have evaluated the outcomes of these patients after curative intent ablation $(52,53)$.

Retrospective cohort studies of patients with multifocal BCLC A HCC (3 tumors $\leq 3 \mathrm{~cm}$ ) reported similar outcomes for patients with 2 tumors as with solitary tumors, with Zhang et al. reporting 1-, 3- and 5-year OS rates of $88.9 \%, 75.5 \%$ and $50.9 \%$ and RFS rates of $85.2 \%, 44.7 \%$ and $24.0 \%$ (52). Outcomes were significantly worse for patients with three tumors, with 1-, 3- and 5-year RFS rates of $76.1 \%, 24.5 \%$ and $2.8 \%$ (52). These findings were consistent with a propensity matched comparison of RFA and resection which found that three tumors and multisegmental disease were independent predictors of poor prognosis (53). Their comparison of treatment modalities found a similar OS but a significantly longer RFS with resection when compared to RFA, with 1-, 3- and 5-year RFS of $87.5 \%, 53.1 \%$, and 20.1 vs. $83.1 \%, 34.0 \%$, and $9.7 \%$, respectively ( $\mathrm{P}=0.001)$ (53). However, resection is not recommended for multifocal HCC by EASL or AASLD guidelines $(3,4)$ and thought 'controversial, but can be considered' by NCCN guidelines (11).

Liver transplant treats both the tumor and the patient's underlying liver disease. Typically these patients fall within the Milan criteria; a single tumor $<5 \mathrm{~cm}$ or $1-3$ tumors each $<3 \mathrm{~cm}$, no macrovascular invasion and without extrahepatic disease; which have consistently shown 5 -year OS of approximately $70 \%$ (9), equivalent outcomes to patients without HCC who receive a transplant $(1,9)$. The main limitation is access, with wait times of over a 1 year for an organ depending on blood type and geographic location. Locoregional therapies play an important role in the management of patients awaiting transplantation through bridging, the treatment of waiting list patients within the transplant criteria, and downstaging, the treatment of patients to reduce tumor burden to within transplant criteria.

\section{Bridging}

Patient awaiting transplantation are considered for bridging therapy to reduce progression and post-transplant recurrence (3). This can include any form of locoregional therapy, with ablation or transarterial embolotherapy most commonly used, with newer data emerging regarding EBRT. It is a particularly important concept given the risk of progression for waiting list patients (7). The AASLD guidelines recommend bridging if an expected waiting time of $\geq 6$ months (3). Difficulty in predicting wait times means that in practice many groups will offer bridging therapies once the patient is listed.

A recent meta-analysis of patients treated with locoregional bridging therapy found dropout rates of $19 \%$ due to all causes and $11 \%$ due to progression (7). For comparative studies, there was a non-significant improvement of dropout rates among patients treated with LRT compared to no therapy for both tumor progression (RR 0.32, 95\% CI: 0.06-1.85) and all causes of dropout (RR $0.38,95 \%$ CI: 0.060-2.370) (7). There was no significant difference in outcomes when the types of locoregional therapy were analyzed. The meta-analysis authors note that the non-randomized nature of these studies could lead to a more aggressive tumor biology in those patients selected for locoregional therapy, possibly explaining the non-significant difference in outcomes (7). There remains debate over the optimal LRT for bridging patients with heterogeneous use amongst centers (4). Single center studies have found an increased time to progression with use of TARE compared to conventional TACE (cTACE) (46,54). Salem et al. (54), in an RCT of unresectable BCLC A or B patients, reported a significantly longer TTP for patients treated with TARE (>26 months) compared to cTACE patients (6.9 months; $\mathrm{P}=0.0012$ ) (HR 0.122, 95\% CI: 0.027-0.557; $\mathrm{P}=0.007$ ). This study is limited by the its early termination due to slow enrollment, after 45 of 179 intended patients were recruited. Other two small RCTs of 24 and 28 patients with unresectable BCLC $\mathrm{A} / \mathrm{B}$ disease did not find a difference in TTP. Pitton et al. reported a median progressionfree survival (PFS) of 180 days for TARE versus 216 days for TACE patients $(\mathrm{P}=0.06193)$ (55), while Kolligs et al. reported a median PFS of 3.6 months for TARE and 3.7 months for TACE (56). These studies are inconclusive and TACE remains more popular in clinical practice but further prospective studies comparing TACE with TARE in the bridging setting would be of interest.

There are few reports on the use of SBRT as bridging therapy in transplant. A planned interim analysis of an RCT involving 69 patients within San Francisco transplant criteria comparing proton beam radiation therapy and TACE showed a trend towards improved 2-year local tumor control ( $88 \%$ vs. $45 \%, \mathrm{P}=0.06)$ and progression-free survival ( $48 \%$ vs. $31 \%, \mathrm{P}=0.06$ ) with proton beam radiotherapy (57). A Toronto group compared TACE, RFA and SBRT in an intention to treat analysis of bridging therapies in 
transplant list patients (58). Patients received SBRT after being deemed poor candidates for another LRT. SBRT patients did have a greater deterioration in liver function than TACE and RFA, $38.9 \%$ vs. $19.4 \%$ vs. $13 \%(\mathrm{P}=0.001)$, respectively; noting that they also had significantly poorer baseline liver function (58). The dropout rates were comparable between therapies; $16.7 \%$ for SBRT vs. $20.2 \%$ for TACE $v$ s. $16.8 \%$ for RFA ( $\mathrm{P}=0.07)$; while posttransplant recurrence rates were higher compared to RFA, $23.3 \%$ for SBRT vs. $30.5 \%$ for TACE vs. $13.3 \%$ for RFA $(\mathrm{P}=0.004)$ (58). They concluded that SBRT may have a role in high-risk patients with impaired liver function, particularly since lower radiation fraction doses can be used with bridging, rather than curative intent (58). Despite these early signs of improved tolerability, the latest NCCN and AASLD guidelines concluded that more prospective and randomized data is needed to determine the safety and efficacy of radiotherapy $(3,11)$. Two phase II randomized control trial are in progress, NCT02182687, a comparison of TACE and SBRT as a bridging option with results due 2022 and NCT02470533, a comparison of TACE using drug-eluting embolics and SBRT in patient ineligible for resection or ablation. Pending those results, EBRT remains an alternative to be considered when those LRT have failed or are contraindicated.

\section{Downstaging}

Downstaging is a controversial topic given the limited supply of organs and the fact that several high-risk factors, microsatellite presence and tumor dedifferentiation, are not available pre-transplant. A meta-analysis of several retrospective studies showed no significant difference in post-transplant outcomes between T3 patients (multifocal tumors with at least one $>5 \mathrm{~cm}$ ) who were downstaged and patients who were transplanted within Milan criteria (7). Their analysis of three studies comparing $\mathrm{T} 3$ patients transplanted after downstaging with $\mathrm{T} 2$ patients did not find a significant difference in post-transplant RFS rates or 3 -year OS rates (RR 1.02) (7). They did show significant increases in 1-year (RR 1.1.7; 95\% CI: 1.01-1.23) and 5-year (RR 1.17; 95\% CI: 1.03-1.32) post-transplant survival rates. However, they rated their quality of evidence as low with a high risk of bias and imprecision (7). That same meta-analysis looked at the outcomes post-transplant between types of downstaging therapies and concluded that there was not enough evidence to decide between modalities (7). These findings are consistent with an earlier meta-analysis by Parikh et al. who found a pooled transplant success rate of $0.48 \%$ (95\% CI: $0.39-0.58$ ) with no significant difference between TACE and TARE (0.48 vs. $0.37 ; \mathrm{P}=0.51)(59)$. Within a heterogeneous group of studies $\left(\mathrm{I}^{2}=84.8 \%\right)$, Parikh et al. found that prospectively designed downstaging protocols outperformed retrospective protocols in transplant success rates (0.68 vs. $0.44 ; \mathrm{P}<0.001)$, probably related to the treatment of more advanced disease in the retrospective protocols (59). The limited studies and data available are reflected in EASL/NCCN/AASLD guidelines, where downstaging 'may be considered' for those outside of transplant criteria, with EASL guidelines adding the proviso that downstaging should be performed within a defined protocol $(3,4,11)$.

Table 1 summarizes outcomes and guidelines for all curative intent approaches.

\section{Palliative intent locoregional therapy for HCC}

\section{TARE}

TARE was initially used in patients who were poor candidates for TACE, e.g., those with vascular invasion who were at high risk of liver failure as a consequence of its embolic effect. The microembolic nature of Y 90 microsphere therapy, due to their small size $(<40 \mu \mathrm{m})$, allowed for it to be used in patients with portal vein occlusion (61). Its popularity has grown as an additional non-curative treatment for patients with BCLC B disease who are not candidates for resection or transplant or BCLC $\mathrm{C}$ patients with locally advanced disease.

In the cohort of patients with unresectable BCLC A or BCLC B tumors; prospective data reports OS outcomes of 16.4-18 months for patients undergoing TARE $(62,63)$. As previously discussed, three small RCTs between TACE and TARE have shown equivalent OS rates (54-56). A metaanalysis of all 3 RCTs did not find a difference in 1 year OS (OR 1.31; 95\% CI: 0.56-3.04) (64). An earlier metaanalysis that included several observational studies but did not include the 2016 RCT by Salem et al. (54) also found equivalent 1-year OS rates. That meta-analysis did find an improved 2- and 3-year OS rates for TARE compared to TACE, $\mathrm{OR}=1.43(1.08-1.89, \mathrm{P}=0.01)$ and $\mathrm{OR}=1.48(1.03-$ $2.13, \mathrm{P}=0.04)$ respectively (50).

With the similarity in OS outcomes, important considerations are given to additional factors such as time to progression, adverse events and quality of life. The discussed RCT data and meta-analyses have shown 
Table 1 Outcomes of curative-intent strategies for various stages and sizes of HCC, with associated guidelines recommendations

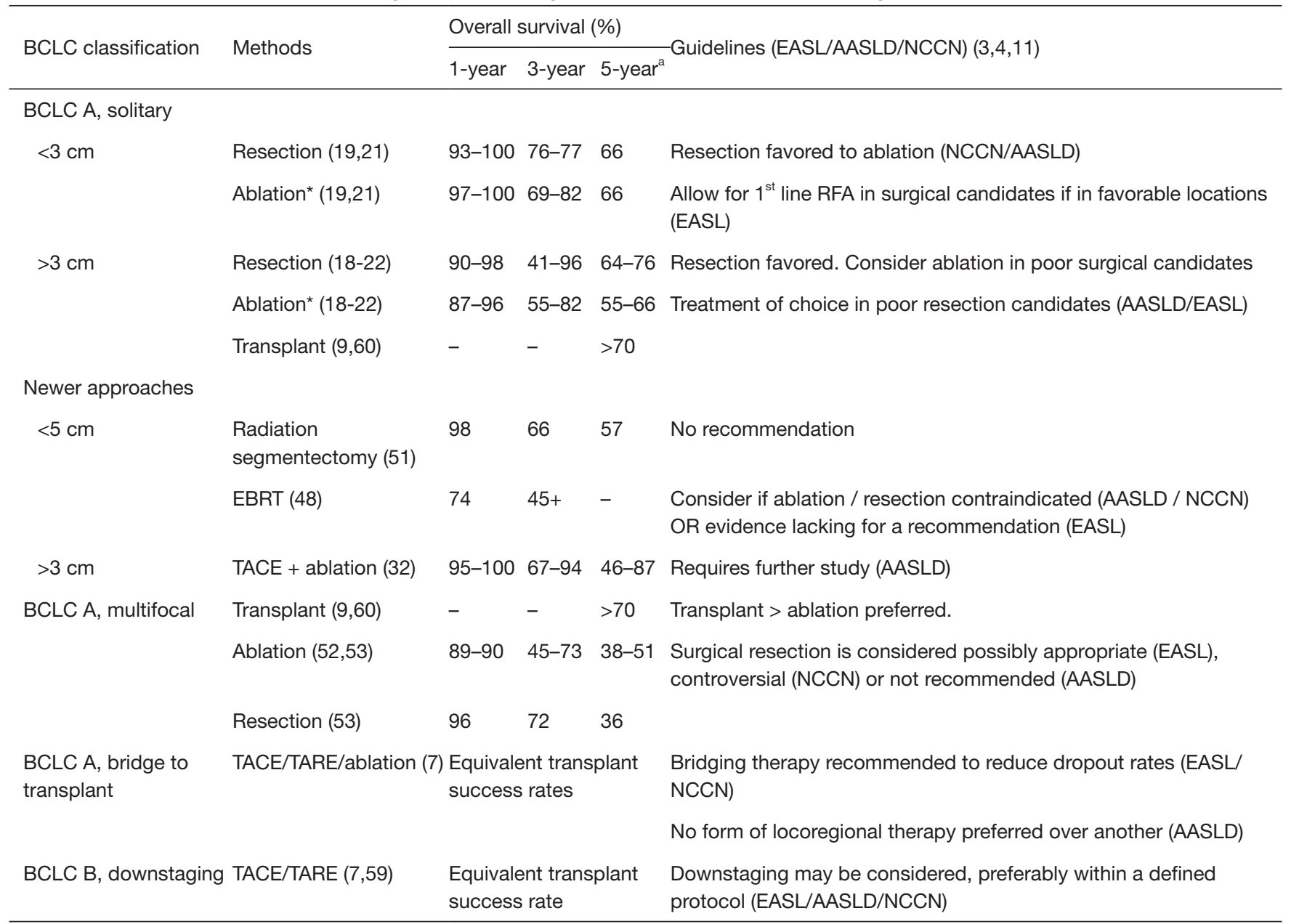

${ }^{a}$, if available. *, RF ablation. MWA considered promising but requiring larger prospective data for safety and efficacy. TACE, transarterial chemoembolization; TARE, transarterial radioembolization; MWA, microwave ablation; HCC, hepatocellular carcinoma; EASL, European Association for the Study of the Liver; AASLD, American Association for the Study of Liver Diseases; NCCN, National Comprehensive Cancer Network.

equivalent safety profiles for both treatments (50,54-56). TACE, however, typically requires repeated treatments to ensure adequate tumor control, as seen in the metaanalysis of 3 RCTs which showed that $80 \%$ of TACE patients required multiple treatments, as opposed to $8 \%$ of TARE patients (64). Kolligs et al. performed the only RCT whose primary endpoint was quality of life using the Functional Assessment of Cancer Therapy-Hepatobiliary (FACT-Hep) questionnaire. Baseline quality of life measures were worse in the TARE patients and remained worse until 12 weeks post-procedure. After this, FACThep scores between the groups were similar (56). These results mimic an earlier prospective non-randomized study that also reported equivalent FACT-Hep outcomes but noted that several sub-features of quality of life, functional wellbeing, social wellbeing and embolotherapy related scores; were improved in the TARE population (65). The longer time to progression, reduced number of treatments and potential for improved quality of life outcomes have encouraged the study of TARE outcomes in this population. Currently, established guidelines note that TACE has a more established evidence base for unresectable BCLC A and BCLC B patients but consider TARE an option of locoregional therapy $(3,4,11)$. Further comparisons are required to discover potential OS differences.

Patients may either present with advanced (BCLC C) 
HCC or stage migrate from an intermediate (BCLC B) category after locoregional therapy. Systemic therapies, firstly sorafenib, have been the standard of care since a modest OS benefit was shown in SHARP trial (66). The AASLD note that this trial and subsequent phase 3 trials that showed an OS benefit for systemic therapies have been limited to patients with a good PS (ECOG <2), Child-Pugh A liver disease, and otherwise adequate organ function (3). There has been interest in the use of TARE in patients with advanced HCC after retrospective studies showed a potential efficacy (67).

Two RCTs, SorAfenib Versus Radioembolization in Advanced Hepatocellular Carcinoma (SARAH) and Selective Internal Radiation therapy $\mathrm{V}$ sorafeNIB (SIRveNIB), have since compared the efficacy and safety of patients treated with TARE $v s$. sorafenib and failed to find a statistically significant superiority difference in OS $(68,69)$. SARAH analyzed 459 patients with locally advanced HCC in a European population and did not find a significant difference in survival; 8.0 months for TARE compared to 9.9 months for sorafenib $(\mathrm{P}=0.18)$ (68). Importantly, in this trial TARE was a 2nd-line liver-directed therapy after failure of TACE. SIRveNIB similarly did not show a significant OS benefit in their study of 360 patients in an Asian population; 10.0 months for TARE compared to 8.8 months for sorafenib $(\mathrm{P}=0.36)$. Regarding adverse events, the SIRveNIB trial comparing resin microspheres with sorafenib and found that significantly fewer grade $\geq 3$ adverse events [36/130 (27.7\%) vs. 82/162 (50.6\%); $\mathrm{P}=0.001]$. SARAH reported similar findings as double the amount of treatment-related serious adverse events were attributed to sorafenib as to TARE. These are important considerations as many patients will discontinue sorafenib therapy due to issues with side effects and tolerability is key in palliative patients with short lifespans. Despite these potential advantages, an OS equivalence must be proven first in RCT as neither SIRveNIB nor SARAH trials were powered for non-inferiority so equivalent OS rates cannot be assumed (70). Of interest are two ongoing RCTs (SORAMIC and STOP-HCC) comparing the use of TARE followed by sorafenib vs. sorafenib alone which will help to define the role of TARE in this challenging patient population.

\section{TACE}

TACE developed circa 1980 in Japan using an emulsion of Lipiodol with an anthracycline chemotherapeutic drug
(60,71-74). Lipiodol is an ethyl ester of iodized fatty acids of poppy seed oil (Guerbet, Aulnay, France). TACE using a Lipiodol drug emulsion followed by a solid embolic became the standard treatment for intermediate stage HCC without portal vein thrombosis as a result of two randomized studies which used either doxorubicin (75) or cis-platinum (76) mixed with Lipiodol, followed by administration of an embolic agent. To date these studies represent the only controlled trials for intra-arterial therapies for HCC demonstrating the superiority of Lipiodol-TACE to best supportive care. Modern controlled trials report OSs after TACE even better than these original RCT's from 2002. The largest TACE RCT ever performed, a phase III study of brivanib as adjuvant therapy to TACE in patients with unresectable HCC in 502 patients, had a median OS in the placebo arm (TACE only) and the brivanib + TACE arm of 26 months (77). A prospective Japan-Korea cooperative study of 99 patients (81\% of whom in Child class A and $87 \%$ with ECOG performance status of 0 ) reported a median OS of 37 months (78). A randomized study comparing Lipiodol TACE and drug-eluting embolic TACE reported a median OS of 28 and 29 months, respectively (79). Hence current expectation is that palliative TACE in early and intermediate HCC should provide survival on the order of 2-3 years.

TACE is also employed in patients with more advanced HCC, such as macrovascular invasion or limited extrahepatic disease in the setting of adequately preserved hepatic function, but survival benefit in this population has not been evaluated in a randomized trial. In a prospective non randomized study, 164 HCC patients with segmental or subsegmental portal vein thrombosis were treated with TACE or conservative care according to the patient's preference after counseling from a tumor board. The 12and 24-month OS rates for the TACE and conservative groups were $30.9 \%, 9.2 \%$, vs. $3.8 \%, 0 \%(\mathrm{P}<0.001)(80)$.

An alternative platform to Lipiodol emulsion for delivery of chemotherapeutics to tumors is polymeric microspheres capable of carrying a drug payload. Several now exist commercially, mostly based on formulations of polyvinyl alcohol. Because microspheres cannot cross the peribiliary capillary plexus, they lodge in the distal arterioles where the payload elutes from the polymer and distributes locally via diffusion. Most of the clinical data with drug-eluting embolics involves doxorubicin-loaded microspheres used to treat HCC. Three randomized trials have shown no oncologic benefit of the drug-eluting embolic platform over Lipiodol TACE or bland embolization with unloaded 
microspheres, with nearly identical PFS and OS in the bland trials (81-83). Therefore Lipiodol-TACE remains the current standard of care in in terminational guidelines for large or multinodular tumor isolated to the liver with preserved liver function and absence of portal vein invasion.

Of greater concern with drug-eluting embolics are toxicity and safety issues. The most concentrated drug release is adjacent to the microspheres at the level of the peribiliary capillary plexus, where the doxorubicin causes local coagulation necrosis of the surrounding hepatic parenchyma, a phenomenon not seen with bland embolics (84). The consequence of this off-target drug delivery is an increased incidence of hepatobiliary injury with doxorubicin-loaded embolics that has been reported following chemoembolization of HCC and NET metastases (85-87). Furthermore, the lack of radiographic conspicuity of drug-eluting embolics results in a higher incidence of non-target embolization than with Lipiodol TACE. A series of 237 cases reported a rate of injury to the gallbladder, stomach, pancreas, and other extra hepatic sites between $5-10 \%$, in excess of the Society of Interventional Radiology Quality Assurance Guidelines for TACE (88). The lack of efficacy and unacceptable toxicity of doxorubicin-loads microspheres is sufficiently well evidenced to preclude their use in clinical practice.

\section{Conclusions}

The disproportionate mortality rate for HCC relative to its prevalence shows the progress that is needed in curative intent therapeutic options. The underlying cirrhotic liver plays a large role in limiting treatment options. Complex treatment decisions are required to decide the optimal treatment method when considering the tumor size, location and underlying liver cirrhosis. Locoregional therapies such as ablation and portal vein embolization have an established role in treating or preparing patients for treatment. New and improving techniques, EBRT, radiation segmentectomy or lobectomy, and combination therapies; show promise but require further prospective data before they can be integrated into treatment algorithms. For palliative-intent liver-directs therapy, Lipiodol TACE remains the standard of practice; to date newer technologies have not yet proven to improve outcomes.

\section{Acknowledgments}

Funding: None.

\section{Footnote}

Provenance and Peer Review: This article was commissioned by the Guest Editors (Mehmet Akce and Shishir K. Maithel) for the series "Hepatocellular Carcinoma" published in Chinese Clinical Oncology. The article was sent for external peer review organized by the Guest Editors and the editorial office.

Conflicts of Interest: All authors have completed the ICMJE uniform disclosure form (available at http://dx.doi. org/10.21037/cco-20-51). The series "Hepatocellular Carcinoma" was commissioned by the editorial office without any funding or sponsorship. MCS reports grants and personal fees from Guerbet LLC, grants from BTG International, grants and personal fees from Sirtex Medical, personal fees from Instylla, personal fees from Genentech, outside the submitted work. The other authors have no other conflicts of interest to declare.

Ethical Statement: The authors are accountable for all aspects of the work in ensuring that questions related to the accuracy or integrity of any part of the work are appropriately investigated and resolved.

Open Access Statement: This is an Open Access article distributed in accordance with the Creative Commons Attribution-NonCommercial-NoDerivs 4.0 International License (CC BY-NC-ND 4.0), which permits the noncommercial replication and distribution of the article with the strict proviso that no changes or edits are made and the original work is properly cited (including links to both the formal publication through the relevant DOI and the license). See: https://creativecommons.org/licenses/by-nc-nd/4.0/.

\section{References}

1. Forner A, Reig M, Bruix J. Hepatocellular carcinoma. Lancet 2018;391:1301-14.

2. Siegel RL, Miller KD, Jemal A. Cancer statistics, 2017. CA Cancer J Clin 2017;67:7-30.

3. Marrero JA, Kulik LM, Sirlin CB, et al. Diagnosis, Staging, and Management of Hepatocellular Carcinoma: 2018 Practice Guidance by the American Association for the Study of Liver Diseases. Hepatology 2018;68:723-50.

4. European Association for the Study of the Liver; European Association for the Study of the Liver. EASL Clinical Practice Guidelines: Management of hepatocellular 
carcinoma. J Hepatol 2018;69:182-236.

5. Yau T, Tang VYF, Yao TJ, et al. Development of Hong Kong Liver Cancer staging system with treatment stratification for patients with hepatocellular carcinoma. Gastroenterology 2014;146:1691-1700.e3.

6. Heckman JT, Devera MB, Marsh JW, et al. Bridging locoregional therapy for hepatocellular carcinoma prior to liver transplantation. Ann Surg Oncol 2008;15:3169-77.

7. Kulik L, Heimbach JK, Zaiem F, et al. Therapies for Patients With Hepatocellular Carcinoma Awaiting Liver Transplantation: A Systematic Review and Meta-analysis. Hepatology 2018;67:381-400.

8. Breen DJ, Lencioni R. Image-guided ablation of primary liver and renal tumours. Vol 12, Nature Reviews Clinical Oncology. Nat Rev Clin Oncol 2015;12:175-86.

9. Mazzaferro V, Regalia E, Doci R, et al. Liver transplantation for the treatment of small hepatocellular carcinomas in patients with cirrhosis. N Engl J Med 1996;334:693-9.

10. Tabrizian P, Jibara G, Shrager B, et al. Recurrence of Hepatocellular Cancer After Resection. Ann Surg 2015;261:947-55.

11. Benson AB, Abbott DE, Abrams TA, et al. NCCN Guidelines Version 3. Hepatobiliary Cancers, 2019.

12. Roayaie S, Obeidat K, Sposito C, et al. Resection of hepatocellular cancer $\leq 2 \mathrm{~cm}$ : Results from two Western centers. Hepatology 2013;57:1426-35.

13. Sasaki A, Kai S, Iwashita Y, et al. Microsatellite distribution and indication for locoregional therapy in small hepatocellular carcinoma. Cancer 2005;103:299-306.

14. Ferrer-Fàbrega J, Forner A, Liccioni A, et al. Prospective validation of ab initio liver transplantation in hepatocellular carcinoma upon detection of risk factors for recurrence after resection. Hepatology 2016;63:839-49.

15. Orlando A, Leandro G, Olivo M, et al. Radiofrequency thermal ablation vs. percutaneous ethanol injection for small hepatocellular carcinoma in cirrhosis: meta-analysis of randomized controlled trials. Am J Gastroenterol 2009; 104:514-24.

16. Majumdar A, Roccarina D, Thorburn D, et al. Management of people with early- or very early-stage hepatocellular carcinoma: An Attempted Network MetaAnalysis. Cochrane Database Syst Rev 2017;3:CD011650.

17. Xu XL, Liu XD, Liang M, et al. Radiofrequency Ablation versus Hepatic Resection for Small Hepatocellular Carcinoma: Systematic Review of Randomized Controlled Trials with Meta-Analysis and Trial Sequential Analysis. Radiology 2018;287:461-72.
18. Huang J, Yan L, Cheng Z, et al. A Randomized Trial Comparing Radiofrequency Ablation and Surgical Resection for HCC Conforming to the Milan Criteria. Ann Surg 2010;252:903-12.

19. Ng KKC, Chok KSH, Chan ACY, et al. Randomized clinical trial of hepatic resection versus radiofrequency ablation for early-stage hepatocellular carcinoma. Br J Surg 2017;104:1775-84.

20. Feng K, Yan J, Li X, et al. A randomized controlled trial of radiofrequency ablation and surgical resection in the treatment of small hepatocellular carcinoma. J Hepatol 2012;57:794-802.

21. Fang Y, Chen W, Liang X, et al. Comparison of long-term effectiveness and complications of radiofrequency ablation with hepatectomy for small hepatocellular carcinoma. J Gastroenterol Hepatol 2014;29:193-200.

22. Chen MS, Li JQ, Zheng Y, et al. A prospective randomized trial comparing percutaneous local ablative therapy and partial hepatectomy for small hepatocellular carcinoma. Ann Surg 2006;243:321-8.

23. Tan W, Deng Q, Lin S, et al. Comparison of microwave ablation and radiofrequency ablation for hepatocellular carcinoma: a systematic review and meta-analysis. Int J Hyperthermia 2019;36:264-72.

24. Chinnaratha MA, Chuang MA, Fraser RJL, et al. Percutaneous thermal ablation for primary hepatocellular carcinoma: A systematic review and meta-analysis. J Gastroenterol Hepatol 2016;31:294-301.

25. Facciorusso A, Di Maso M, Muscatiello N. Microwave ablation versus radiofrequency ablation for the treatment of hepatocellular carcinoma: A systematic review and meta-analysis. Int J Hyperthermia 2016;32:339-44.

26. Lee GC, Ferrone CR, Vagefi PA, et al. Surgical resection versus ablation for early-stage hepatocellular carcinoma: A retrospective cohort analysis. Am J Surg 2019;218:157-63.

27. Nakazawa T, Kokubu S, Shibuya A, et al. Radiofrequency ablation of hepatocellular carcinoma: Correlation between local tumor progression after ablation and ablative margin. AJR Am J Roentgenol 2007;188:480-8.

28. Glassberg MB, Ghosh S, Clymer JW, et al. Microwave ablation compared with hepatic resection for the treatment of hepatocellular carcinoma and liver metastases: a systematic review and meta-analysis. World J Surg Oncol 2019; 17:98.

29. Wang X, Hu Y, Ren M, et al. Efficacy and Safety of Radiofrequency Ablation Combined with Transcatheter Arterial Chemoembolization for Hepatocellular Carcinomas Compared with Radiofrequency Ablation 
Alone: A Time-to-Event Meta-Analysis. Korean J Radiol 2016;17:93-102.

30. Chen QW, Ying HF, Gao S, et al. Radiofrequency ablation plus chemoembolization versus radiofrequency ablation alone for hepatocellular carcinoma: A systematic review and meta-analysis. Clin Res Hepatol Gastroenterol 2016;40:309-14.

31. Peng ZW, Zhang YJ, Chen MS, et al. Radiofrequency ablation with or without transcatheter arterial chemoembolization in the treatment of hepatocellular carcinoma: a prospective randomized trial. J Clin Oncol 2013;31:426-32.

32. Wang W, Sinan H, Xu L. Radiofrequency ablation combined with transcatheter arterial chemoembolization therapy versus surgical resection for Barcelona-Clinic Liver Cancer (BCLC) A hepatocellular carcinoma: a metaanalysis. J Interv Med 2018;1:49-57.

33. Sheta E, El-Kalla F, El-Gharib M, et al. Comparison of single-session transarterial chemoembolization combined with microwave ablation or radiofrequency ablation in the treatment of hepatocellular carcinoma: A randomized-controlled study. Eur J Gastroenterol Hepatol 2016;28:1198-203.

34. Ginsburg M, Zivin SP, Wroblewski K, et al. Comparison of combination therapies in the management of hepatocellular carcinoma: Transarterial chemoembolization with radiofrequency ablation versus microwave ablation. J Vasc Interv Radiol 2015;26:330-41.

35. Hu H, Chen GF, Yuan W, et al. Microwave ablation with chemoembolization for large hepatocellular carcinoma in patients with cirrhosis. Int J Hyperthermia 2018;34:1351-8.

36. Lencioni R, de Baere T, Martin RC, et al. Image-Guided Ablation of Malignant Liver Tumors: Recommendations for Clinical Validation of Novel Thermal and NonThermal Technologies - A Western Perspective. Liver Cancer 2015;4:208-14.

37. Lu DSK, Raman SS, Limanond P, et al. Influence of large peritumoral vessels on outcome of radiofrequency ablation of liver tumors. J Vasc Interv Radiol 2003;14:1267-74.

38. Lu DSK, Yu NC, Raman SS, et al. Radiofrequency Ablation of Hepatocellular Carcinoma: Treatment Success as Defined by Histologic Examination of the Explanted Liver. Radiology 2005;234:954-60.

39. Brace CL, Laeseke PF, Sampson LA, et al. Microwave ablation with a single small-gauge triaxial antenna: in vivo porcine liver model. Radiology 2007;242:435-40.

40. de Baere T, Bessoud B, Dromain C, et al. Percutaneous radiofrequency ablation of hepatic tumors during temporary venous occlusion. AJR Am J Roentgenol 2002;178:53-9.

41. Berzigotti A, Reig M, Abraldes JG, et al. Portal hypertension and the outcome of surgery for hepatocellular carcinoma in compensated cirrhosis: A systematic review and meta-analysis. Hepatology 2015;61:526-36.

42. Abulkhir A, Limongelli P, Healey AJ, et al. Preoperative Portal Vein Embolization for Major Liver Resection. Ann Surg 2008;247:49-57.

43. Garlipp B, de Baere T, Damm R, et al. Left-liver hypertrophy after therapeutic right-liver radioembolization is substantial but less than after portal vein embolization. Hepatology 2014;59:1864-73.

44. Hayashi S, Baba Y, Ueno K, et al. Acceleration of primary liver tumor growth rate in embolized hepatic lobe after portal vein embolization. Acta radiol 2007;48:721-7.

45. Gabr A, Polineni P, Mouli SK, et al. Neoadjuvant Radiation Lobectomy As an Alternative to Portal Vein Embolization in Hepatocellular Carcinoma. Semin Nucl Med 2019;49:197-203.

46. Vouche M, Lewandowski RJ, Atassi R, et al. Radiation lobectomy: Time-dependent analysis of future liver remnant volume in unresectable liver cancer as a bridge to resection. J Hepatol 2013;59:1029-36.

47. Kalogeridi MA, Zygogianni A, Kyrgias G, et al. Role of radiotherapy in the management of hepatocellular carcinoma: A systematic review. World J Hepatol 2015;7:101-12.

48. Wahl DR, Stenmark MH, Tao Y, et al. Outcomes After Stereotactic Body Radiotherapy or Radiofrequency Ablation for Hepatocellular Carcinoma. J Clin Oncol 2016;34:452-9.

49. Berger NG, Tanious MN, Hammad AY, et al. External radiation or ablation for solitary hepatocellular carcinoma: A survival analysis of the SEER database. J Surg Oncol 2017;116:307-12.

50. Facciorusso A, Serviddio G, Muscatiello N. Transarterial radioembolization vs chemoembolization for hepatocarcinoma patients: A systematic review and metaanalysis. World J Hepatol 2016;8:770-8.

51. Lewandowski RJ, Gabr A, Abouchaleh N, et al. Radiation Segmentectomy: Potential Curative Therapy for Early Hepatocellular Carcinoma. Radiology 2018;287:1050-8.

52. Zhang W, Jiang L, Yan L, et al. Radiofrequency ablation for HCC patients with multifocal tumours meeting the Milan criteria: A single-centre experience. Dig Liver Dis 2016;48:1485-91.

53. Jiang L, Yan L, Wen T, et al. Comparison of Outcomes 
of Hepatic Resection and Radiofrequency Ablation for Hepatocellular Carcinoma Patients with Multifocal Tumors Meeting the Barcelona-Clinic Liver Cancer Stage A Classification. J Am Coll Surg 2015;221:951-61.

54. Salem R, Gordon AC, Mouli S, et al. Y90

Radioembolization Significantly Prolongs Time to Progression Compared With Chemoembolization in Patients With Hepatocellular Carcinoma.

Gastroenterology 2016;151:1155-1163.e2.

55. Pitton MB, Kloeckner R, Ruckes C, et al. Randomized Comparison of Selective Internal Radiotherapy (SIRT) Versus Drug-Eluting Bead Transarterial Chemoembolization (DEB-TACE) for the Treatment of Hepatocellular Carcinoma. Cardiovasc Intervent Radiol 2015;38:352-60.

56. Kolligs FT, Bilbao JI, Jakobs T, et al. Pilot randomized trial of selective internal radiation therapy vs. chemoembolization in unresectable hepatocellular carcinoma. Liver Int 2015;35:1715-21.

57. Bush DA, Smith JC, Slater JD, et al. Randomized Clinical Trial Comparing Proton Beam Radiation Therapy with Transarterial Chemoembolization for Hepatocellular Carcinoma: Results of an Interim Analysis. Int J Radiat Oncol Biol Phys 2016;95:477-82.

58. Sapisochin G, Barry A, Doherty M, et al. Stereotactic body radiotherapy vs. TACE or RFA as a bridge to transplant in patients with hepatocellular carcinoma. An intention-totreat analysis. J Hepatol 2017;67:92-9.

59. Parikh ND, Waljee AK, Singal AG. Downstaging hepatocellular carcinoma: A systematic review and pooled analysis. Liver Transpl 2015;21:1142-52.

60. Konno T, Maeda H, Iwai K, et al. Effect of arterial administration of high-molecular-weight anticancer agent SMANCS with lipid lymphographic agent on hepatoma: a preliminary report. Eur J Cancer Clin Oncol 1983;19:1053-65.

61. Abouchaleh N, Gabr A, Ali R, et al. 90 Y radioembolization for locally advanced hepatocellular carcinoma with portal vein thrombosis: Long-term outcomes in a 185 -patient cohort. J Nucl Med 2018;59:1042-8.

62. Salem R, Lewandowski RJ, Kulik L, et al. Radioembolization results in longer time-to-progression and reduced toxicity compared with chemoembolization in patients with hepatocellular carcinoma. Gastroenterology 2011;140:497-507.e2.

63. Moreno-Luna LE, Yang JD, Sanchez W, et al. Efficacy and Safety of Transarterial Radioembolization Versus Chemoembolization in Patients With Hepatocellular
Carcinoma. Cardiovasc Intervent Radiol 2013;36:714-23.

64. Casadei Gardini A, Tamburini E, Iñarrairaegui M, et al. Radioembolization versus chemoembolization for unresectable hepatocellular carcinoma: A meta-analysis of randomized trials. Onco Targets Ther 2018;11:7315-21.

65. Salem R, Gilbertsen M, Butt Z, et al. Increased quality of life among hepatocellular carcinoma patients treated with radioembolization, compared with chemoembolization. Clin Gastroenterol Hepatol 2013;11:1358-1365.e1.

66. Llovet JM, Ricci S, Mazzaferro V, et al. Sorafenib in Advanced Hepatocellular Carcinoma. N Engl J Med 2008;359:378-90.

67. Gramenzi A, Golfieri R, Mosconi C, et al. Yttrium-90 radioembolization vs sorafenib for intermediate-locally advanced hepatocellular carcinoma: a cohort study with propensity score analysis. Liver Int 2015;35:1036-47.

68. Vilgrain V, Pereira H, Assenat E, et al. Efficacy and safety of selective internal radiotherapy with yttrium-90 resin microspheres compared with sorafenib in locally advanced and inoperable hepatocellular carcinoma (SARAH): an open-label randomised controlled phase 3 trial. Lancet Oncol 2017;18:1624-36.

69. Chow PKH, Gandhi M, Tan SB, et al. SIRveNIB: Selective internal radiation therapy versus sorafenib in Asia-Pacific patients with hepatocellular carcinoma. J Clin Oncol 2018;36:1913-21.

70. Llovet JM, Finn RS. Negative phase 3 study of $90 \mathrm{Y}$ microspheres versus sorafenib in HCC. Lancet Oncol 2018;19:e69.

71. Nakamura H, Hashimoto T, Oi H, Sawada S. Transcatheter oily chemoembolization of hepatocellular carcinoma. Radiology 1989;170:783-6.

72. Uchida H, Ohishi H, Matsuo N, et al. Transcatheter hepatic segmental arterial embolization using lipiodol mixed with an anticancer drug and Gelfoam particles for hepatocellular carcinoma. Cardiovasc Intervent Radiol 1990;13:140-5.

73. Yamada R, Sato M, Kawabata M, et al. Hepatic artery embolization in 120 patients with unresectable hepatoma. Radiology 1983;148:397-401.

74. Matsui O, Kadoya M, Yoshikawa J, et al. Small hepatocellular carcinoma: treatement with subsegmental transcatheter arterial embolization. Radiology 1993;188:79-83.

75. Llovet JM, Real MI, Montana X, et al. Arterial embolisation or chemoembolisation versus symptomatic treatment in patients with unresectable hepatocellular carcinoma: a randomised controlled trial. Lancet 
2002;359:1734-9.

76. Lo CM, Ngan H, Tso WK, et al. Randomized controlled trial of transarterial lipiodol chemoembolization for unresectable hepatocellular carcinoma. Hepatology 2002;35:1164-71.

77. Kudo M, Han G, Finn RS, et al. Brivanib as adjuvant therapy to transarterial chemoembolization in patients with hepatocellular carcinoma: a randomized phase III trial. Hepatology 2014;60:1697-707.

78. Ikeda M, Arai Y, Park SJ, et al. Prospective study of transcatheter arterial chemoembolization for unresectable hepatocellular carcinoma: an Asian cooperative study between Japan and Korea. J Vasc Interv Radiol 2013;24:490-500.

79. Golfieri R, Giampalma E, Renzulli M, et al. Randomised controlled trial of doxorubicineluting beads vs conventional chemoembolisation for hepatocellular carcinoma. Br J Cancer 2014;111:255-64.

80. Luo J, Guo RP, Lai EC, et al. Transarterial chemoembolization for unresectable hepatocellular carcinoma with portal vein tumor thrombosis: a prospective comparative study. Ann Surg Oncol 2011;18:413-20.

81. Lammer J, Malgari K, Vogo T, et al. Prospective randomized study of doxorubicin-eluting -bead embolization in the treatment of hepatocellular carcinoma: results of the PRECISION V study. Cardiovasc Intervent Radiol 2010;33:41-52.

82. Malagari K, Pomoni M, Kelekis A, et al. Prospective randomized comparison of chemoembolization with

Cite this article as: O'Leary C, Mahler M, Soulen MC. Liverdirected therapy for hepatocellular carcinoma. Chin Clin Oncol 2021;10(1):8. doi: 10.21037/cco-20-51 doxorubicin-eluting beads and bland embolization with BeadBlock for hepatocellular carcinoma. Cardiovasc Intervent Radiol 2010;33:541-51.

83. Brown KT, Do RK, Gonen M, et al. Randomized Trial of Hepatic Artery Embolization for Hepatocellular Carcinoma Using Doxorubicin-Eluting Microspheres Compared With Embolization With Microspheres Alone. J Clin Oncol 2016;34:2046-53.

84. Namur J, Wassef M, Mollot JM, et al. Drug-eluting beards for liver embolization: concentration of doxorubicin in tissue and in beads in a pig model. J Vasc Interv Radiol 2010;21:259-67.

85. Monier A, Guiu B, Duran R, et al. Liver and biliary damages following trans arterial chemoembolization of hepatocellular carcinoma: comparison between drug-eluting beads and lipiodol emulsion. Eur Radiol 2017;27:1431-9.

86. Bhagat N, Reyes DK, Lin M, et al. Phase II study of chemoembolization with drug-eluting beads in patients with hepatic neuroendocrine metastases: high incidence of biliary injury. Cardiovasc Intervent Radiol 2013; 36:449-59.

87. Guiu B, Deschamps F, Aho S, et al. Liver/biliary injuries following chemoembolisation of endocrine tumours and hepatocellular carcinoma: lipiodol vs. drug-eluting beads. J Hepatol 2012;56:609-17.

88. Malagari K, Pomoni M, Spyridopoulos TN, et al. Safety profile of sequential transcatheter chemoembolization with DC Bead ${ }^{\mathrm{TM}}$ : results of 237 hepatocellular carcinoma (HCC) patients. Cardiovasc Intervent Radiol 2011;34:774-85. 